\title{
Hydromagnetic and Soret driving free convection in an inclined porous cavity saturated by a conducting binary mixture
}

\author{
A. Rtibi, M. Hasnaoui and A. Amahmid \\ Cadi Ayyad University, Faculty of Sciences Semlalia, LMFE, Unit affiliated to CNRST (URAC 27) \\ BP 2390, Marrakech, Morocco
}

\begin{abstract}
We consider the Soret convection in an inclined rectangular Darcy porous medium filled with an electrically conductive binary fluid. The long sidewalls of the porous cavity are subject to constant gradients of heat and submitted to a uniform and constant transversal magnetic field while its short sides are adiabatic and impermeable. An approximate analytical solution to the present problem, valid in the central part of the shallow cavity, is obtained on the basis of the parallel flow approximation and validated numerically using a finite-difference method. Results are presented in terms of streamlines, isotherms, iso-concentration lines, Nusselt and Sherwood numbers and separation of species for Hartmann number varying in the range [0, 20]. A good agreement is observed between the analytical predictions and numerical simulations.
\end{abstract}

\section{Introduction}

Because of its strong involvement in many engineering applications, Soret-convection in rectangular porous cavities has received a growing attention through the decades. Most of the existing studies are concerned with Soret driven thermosolutal convection within porous cavities saturated by electrically neutral fluids which is the case in most practical situations [1-3]. However, the problem of natural convection of an electrically conducting fluid in the presence of an applied magnetic field is also of considerable interest from the viewpoint of many applications; especially in metal working processes or in the separation of molten metals from non-metallic inclusions by the application of a magnetic field [4]. Also, the use of liquid metals as a coolant medium, manufacturing crystals and metallurgical melting are examples involving the interaction between magnetic fields and convection [5]. In general, when an electrically conducting fluid is subjected to a magnetic field, the fluid motion induces an electric current and the fluid velocity is reduced due to the interaction between the electric current and the motion [6]. Earlier, Raptis and Vlahos [7] have considered free convection flows of a conducting fluid within a porous medium bounded by two horizontal plates. More recently, combined effects of an applied magnetic field and an external shear stress on Soret natural convection developed in a horizontal porous layer heated from below with a constant heat flux was studied analytically and numerically by Bourich et al. [8]. It is reported in this study that the introduction of magnetic buoyancy forces has a stabilizing effect on the system; it leads to a reduction of the flow intensity and heat transfer. However, it can engender an increase or a reduction of the mass transfer dependently on the values of the Hartmann number and the separation ratio.

The problem of laminar hydromagnetic doublediffusive natural convection flows within inclined rectangular porous cavity in the presence of Soret effect has not been considered in the literature despite the fact that it is of interest both fundamentally and practically. The main objective of this study is the examination of the Hartmann number effect on the flow intensity, heat and mass transfer characteristics and separation of species.

\section{Mathematical formulation}

The configuration under study is sketched in Fig. 1. It consists of a tall rectangular porous cavity of width $\mathrm{H}^{\prime}$, height $\mathrm{L}^{\prime}$ and impermeable boundaries. The porous matrix is saturated with an electrically conducting binary fluid and inclined at an angle $\theta$ with respect to the horizontal. A uniform magnetic field of strength B is applied normally to the long sides and the latter are also submitted to uniform fluxes of heat while the short sides are insulated. The mixture saturating the porous medium is assumed homogeneous, isotropic and modelled as a Boussinesq incompressible fluid whose density varies linearly with temperature and concentration as $\rho=\rho_{0}\left[1-\beta_{T}\left(T^{\prime}-T_{0}^{\prime}\right)-\beta_{S}\left(S^{\prime}-S_{0}^{\prime}\right)\right]$. The remaining physical properties are considered constant. Using the Darcy model and taking into account the Soret effect, the dimensionless governing equations describing the conservation of momentum, energy and species in the saturated porous medium are written as follows: 
$\nabla^{2} \Psi+\mathrm{Ha}^{2} \frac{\partial \mathrm{u}}{\partial \mathrm{y}}=-\mathrm{R}_{\mathrm{T}}\left(\cos \theta \frac{\partial}{\partial \mathrm{x}}-\sin \theta \frac{\partial}{\partial \mathrm{y}}\right)(\mathrm{T}+\varphi \mathrm{S})$

$\frac{\partial \mathrm{T}}{\partial \mathrm{t}}+\mathrm{u} \frac{\partial \mathrm{T}}{\partial \mathrm{x}}+\mathrm{v} \frac{\partial \mathrm{T}}{\partial \mathrm{y}}=\nabla^{2} \mathrm{~T}$

$\varepsilon \frac{\partial S}{\partial t}+u \frac{\partial S}{\partial x}+v \frac{\partial S}{\partial y}=\frac{1}{L e}\left(\nabla^{2} S-\nabla^{2} T\right)$

$\nabla^{2} \Psi=-\zeta$

$\mathrm{u}=\frac{\partial \Psi}{\partial \mathrm{y}}, \mathrm{v}=-\frac{\partial \Psi}{\partial \mathrm{x}}$

In the above equations, $\Psi, \mathrm{T}$ and $\mathrm{S}$ are the dimensionless stream function, temperature and solute concentration, respectively. The boundary conditions associated to the governing equations are:

$$
\begin{aligned}
& \mathrm{y}= \pm 1 / 2: \quad \Psi=0, \quad \frac{\partial \mathrm{T}}{\partial \mathrm{y}}=-1, \quad \frac{\partial \mathrm{S}}{\partial \mathrm{y}}=-1 \\
& \left.\mathrm{x}= \pm \mathrm{A}_{\mathrm{r}} / 2: \Psi=0, \quad \frac{\partial \mathrm{T}}{\partial \mathrm{x}}=0, \quad \frac{\partial \mathrm{S}}{\partial \mathrm{x}}=0\right\}
\end{aligned}
$$

In addition to the inclination $\theta$ of the cavity, the above equations indicate that the present problem is governed by five other dimensionless parameters, which are the separation parameter, $\varphi$, the Hartmann number, Ha, the thermal Darcy-Rayleigh number, $\mathrm{R}_{\mathrm{T}}$, the Lewis number, Le, and the cavity aspect ratio, $\mathrm{A}_{\mathrm{r}}$. These parameters are defined as:

$$
\left.\begin{array}{l}
\varphi=-\frac{\beta_{\mathrm{S}} \mathrm{S}_{0}^{\prime}\left(1-\mathrm{S}_{0}^{\prime}\right) \mathrm{D}_{\mathrm{T}}}{\beta_{\mathrm{T}} \mathrm{D}_{\text {eff }}}, \mathrm{Ha}=\mathrm{B} \sqrt{\gamma \mathrm{K} / \mu} \\
\mathrm{R}_{\mathrm{T}}=\frac{\mathrm{g} \beta_{\mathrm{T}} \mathrm{K} \Delta \mathrm{T}^{\prime} \mathrm{H}^{\prime}}{\alpha v}, \mathrm{Le}=\frac{\alpha}{\mathrm{D}_{\text {eff }}}, \mathrm{A}_{\mathrm{r}}=\frac{\mathrm{L}^{\prime}}{\mathrm{H}^{\prime}}
\end{array}\right\}
$$

Where $D_{\text {eff }}=D \varepsilon^{\prime}$ is the effective mass diffusivity and $\mathrm{D}$ and $\mathrm{D}_{\mathrm{T}}$ are respectively the mass diffusivity and the thermodiffusion coefficient. The heat and solute transfer rates across the layer are evaluated in terms of Nusselt and Sherwood numbers which are defined as:

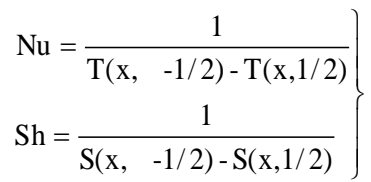

\section{Analytical solution}

The analytical solution is developed for steady state parallel flows, induced in slender cavities $\left(A_{r}>>1\right)$ under specific thermal boundary conditions. A typical behaviour of the parallel flow solution is illustrated numerically in Fig. 2. This behaviour allows the following simplifications:

$$
\left.\begin{array}{l}
\Psi(\mathrm{x}, \mathrm{y})=\Psi(\mathrm{y}) \\
\mathrm{T}(\mathrm{x}, \mathrm{y})=\mathrm{C}_{\mathrm{T}} \mathrm{x}+\theta_{\mathrm{T}}(\mathrm{y}) \\
\mathrm{S}(\mathrm{x}, \mathrm{y})=\mathrm{C}_{\mathrm{S}} \mathrm{x}+\theta_{\mathrm{S}}(\mathrm{y})
\end{array}\right\}
$$

Where $\mathrm{C}_{\mathrm{T}}$ and $\mathrm{C}_{\mathrm{S}}$ are unknown constant temperature and concentration gradients respectively in the $\mathrm{x}$ direction. They are determined by imposing zero heat and mass fluxes across any transversal section of the cavity.

For $\Omega>0$, the solution of the simplified governing equations, satisfying the boundary conditions in the $y$ direction, is obtained as:

$$
\begin{aligned}
& \Psi(y)=-B \Omega \cosh (\Omega y)+G \\
& \mathrm{u}(\mathrm{y})=-\mathrm{B} \Omega^{2} \sinh (\Omega y) \\
& \mathrm{T}(\mathrm{x}, \mathrm{y})=\mathrm{C}_{\mathrm{T}} \mathrm{x}-\mathrm{C}_{\mathrm{T}} \mathrm{B} \sinh (\Omega \mathrm{y})+\left(\mathrm{C}_{\mathrm{T}} \mathrm{G}-1\right) \mathrm{y}(12) \\
& \mathrm{S}(\mathrm{x}, \mathrm{y})=\mathrm{C}_{\mathrm{S}} \mathrm{x}-\left(\mathrm{C}_{\mathrm{T}}+\mathrm{C}_{\mathrm{S}} \mathrm{Le}\right) \mathrm{B} \sinh (\Omega \mathrm{y})+\left(\left(\mathrm{C}_{\mathrm{T}}+\mathrm{C}_{\mathrm{s}} L \mathrm{e}\right) \mathrm{G}-1\right) \mathrm{y}
\end{aligned}
$$

where: $\Omega^{2}=\mathrm{R}_{\mathrm{T}} \sin (\theta)\left[\mathrm{C}_{\mathrm{T}}+\varphi\left(\mathrm{C}_{\mathrm{T}}+\mathrm{C}_{\mathrm{S}} \mathrm{Le}\right)\right] /\left(1+\mathrm{Ha}^{2}\right)$

$\mathrm{G} \Omega^{2}=\mathrm{R}_{\mathrm{T}}\left[\left(\mathrm{C}_{\mathrm{T}}+\varphi \mathrm{C}_{\mathrm{s}}\right) \cos (\theta)+(1+\varphi) \sin (\theta)\right] /\left(1+\mathrm{Ha}^{2}\right)$ and $\Omega \mathrm{B}=\mathrm{G} / \cosh \left(\frac{\Omega}{2}\right)$.

The analytical expressions of $\mathrm{C}_{\mathrm{T}}$ and $\mathrm{C}_{\mathrm{S}}$ are obtained as:

$\mathrm{C}_{\mathrm{T}}=\alpha_{1} \mathrm{~B}^{2} \mathrm{C}_{\mathrm{T}}+\mathrm{B}\left(1 \quad \mathrm{GC}_{\mathrm{T}}\right) \alpha_{2}$

$\left.\mathrm{C}_{\mathrm{S}}=\mathrm{C}_{\mathrm{T}}+\mathrm{Le}\left[\alpha_{1} \mathrm{~B}^{2}\left(\mathrm{LeC}_{\mathrm{s}}+\mathrm{C}_{\mathrm{T}}\right)+\mathrm{B} \alpha_{2}\left(1 \mathrm{G}\left(\mathrm{C}_{\mathrm{T}}+\mathrm{LeC}_{\mathrm{s}}\right)\right)\right]\right\}$

where $\alpha_{1}=\frac{\Omega}{2}[\sinh (\Omega)-\Omega]$ and $\alpha_{2}=\Omega \cosh \left(\frac{\Omega}{2}\right)-2 \sinh \left(\frac{\Omega}{2}\right)$.

Then, using Eqs. (8), (12) and (13), the expressions of $\mathrm{Nu}$ and Sh are obtained as:

$$
\left.\begin{array}{l}
\mathrm{Nu}=\frac{1}{\left(1-\mathrm{BC}_{\mathrm{T}} \alpha_{2}\right)} \\
\mathrm{Sh}=\frac{1}{\left[1-\mathrm{B}\left(\mathrm{C}_{\mathrm{T}}+\mathrm{C}_{\mathrm{S}} \text { Le }\right) \alpha_{2}\right]}
\end{array}\right\}
$$

The system of transcendental Eqs. (14) can be solved simultaneously by using the Newton-Raphson iterative procedure. In this way, the temperature and concentration gradients $\mathrm{C}_{\mathrm{T}}$ and $\mathrm{C}_{\mathrm{S}}$ can be obtained for any combination of $\mathrm{R}_{\mathrm{T}}, \theta$, Le and $\varphi$. The velocity, temperature and concentration profiles are then given by Eqs. (10)-(13) while the Nusselt and Sherwood numbers can be calculated from Eq. (15).

\section{Results and discussions}

The present study is mainly focused on the effect of parameters associated to the applied magnetic field, the Soret effect and the inclination of the cavity. The results obtained are illustrated in terms of $\Psi_{\mathrm{c}}, \mathrm{Nu}, \mathrm{Sh}$ and $\Delta \mathrm{C}$ (defined as the difference of the mass fraction of the species per unit length between the short ends of the cavity) variations versus $\mathrm{Ha}$ (the ratio of electromagnetic force to the viscous force) for $\mathrm{Le}=10, \mathrm{R}_{\mathrm{T}}=200$ and various values of $\theta$ and $\varphi$.

Figs. 3(a)-(d) illustrate the evolutions of the flow intensity, $\Psi_{c}$, the Nusselt number, $\mathrm{Nu}$, the Sherwood number, $\mathrm{Sh}$, and the separation, $\Delta \mathrm{C}$, with the Hartmann number, $\mathrm{Ha}$, for $\mathrm{R}_{\mathrm{T}}=200$, Le $=10, \varphi= \pm 0.5$ and various 
values of $\theta$. The curves presented in these graphs are obtained analytically (solid and dashed lines) and validated numerically (dots) by solving the full governing equations with $A_{r} \geq 8$. As it can be seen in these figures, the magnetic field has a strong effect on the results presented. By increasing $\mathrm{Ha}$, both $\Psi_{\mathrm{c}}$ (Fig. 3a) and $\mathrm{Nu}$ (Fig. 3b) evolutions are characterized by monotonous decreases towards the diffusive regime, indicating that the magnetic field has a negative impact on the flow intensity and heat transfer; it leads to the cancellation of the flow circulation from values of $\mathrm{Ha}$ which are depending on $\theta$ and $\varphi$. Therefore, the magnetic field plays an important damping role (a stabilizing effect on the system) by reducing the flow intensity. In the case of Sh (Fig. 3c), its evolution is characterized by a different behaviour if we except the case $(\theta, \varphi)=\left(135^{\circ}, 0.5\right)$ for which the decrease of Sh (towards the diffusive regime) accompanying the increase of $\mathrm{Ha}$ is monotonous. For the remaining cases, we note the existence of a secondary maximum of $\mathrm{Sh}$ around $\mathrm{Ha}=5$ before the decrease towards the diffusive regime. Fig. 3d illustrates the evolution of the separation $\Delta \mathrm{C}$ versus $\mathrm{Ha}$; the curves exhibit a maximum whose location depends both on $\varphi$ and $\theta$. Note that the values of Ha corresponding to these maxima are located in the interval $8.86 \leq \mathrm{Ha} \leq 15.28$. In this range of $\mathrm{Ha}$, the flow intensity is small but not zero. This means that the coupling between convection and thermodiffusion is optimal in this interval, leading to a maximum of separation.

\section{Conclusion}

The effect of a transverse magnetic field on buoyancydriven convection in an inclined rectangular porous cavity is studied numerically and analytically. It is found that the heat and mass transfer mechanisms and the flow characteristics inside the enclosure are strongly depending on the governing parameters. The stabilizing effect of the magnetic buoyancy forces on the system, characterized by a reduction of the flow intensity and heat transfer, is proved. However, it can engender an increase or a reduction of mass transfer dependently on the combinations of the governing parameters. Finally, optimal combinations of these parameters leading to maximum separation of the species are identified.

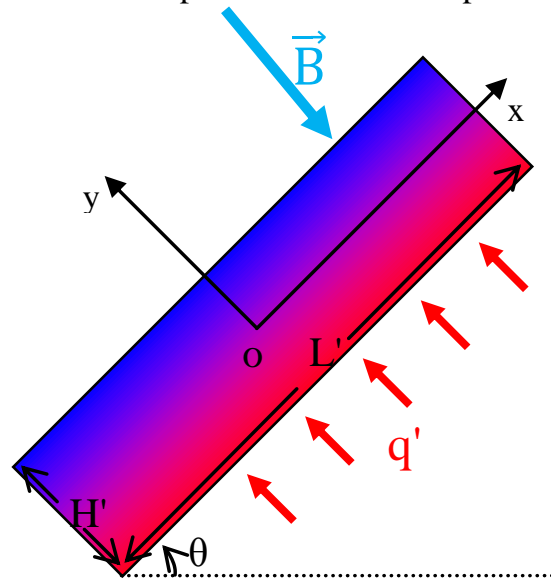

Fig. 1. Schematic diagram of the physical model and coordinate system.
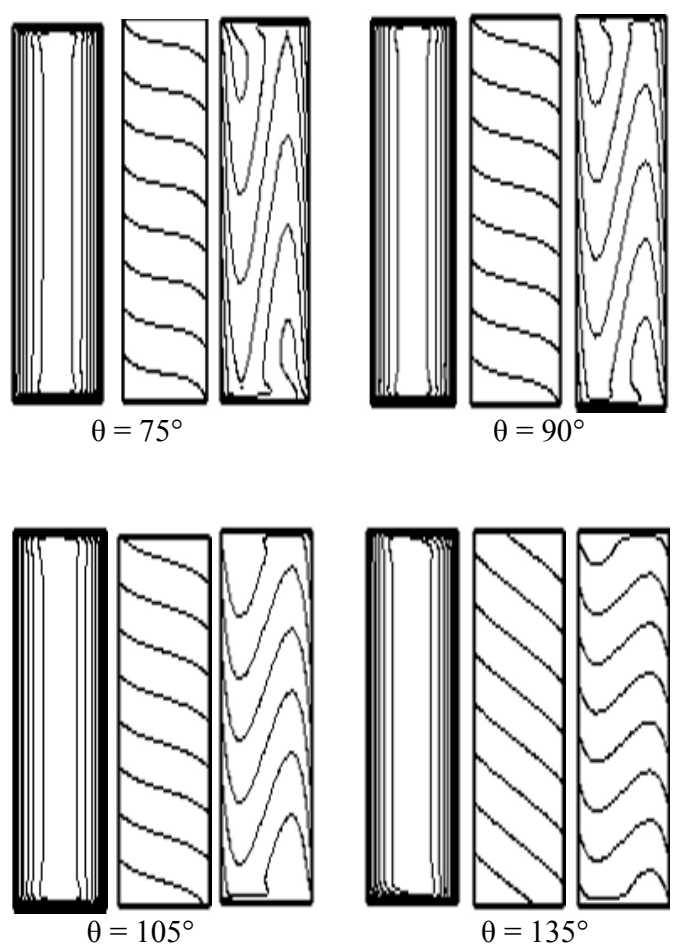

Fig. 2. Streamlines, isotherms and iso-concentrations for $\mathrm{R}_{\mathrm{T}}=200, \mathrm{Le}=10, \mathrm{Ha}=4$ and $\varphi=0.5$.

\section{References}

1. O. Sovran, M. C. Charrier-Mojtabi, and A. Mojtabi, Naissance de la convection thermosolutale en couche poreuse infinie avec effet Soret. C. R. Acad. Sci. Paris, t. 329, Série II b, 287-293, 2001.

2. M. Bourich, M. Hasnaoui, A. Amahmid and M. Mamou, Onset of convection and finite amplitude flow due to Soret effect within a horizontal sparsely packed porous enclosure heated from below, Int. J. Heat Fluid Flow, 25,513-525, 2005.

3. M. Er-Raki, M. Hasnaoui, A. Amahmid and M. Mamou, Soret effect on the boundary layer flow regime in a vertical porous enclosure subject to horizontal heat and mass fluxes. Int. J Heat Mass Transfer, 49, 3111-3120, 2006.

4. K. Ezaki, M. Kaneda, T. Tagawa, and H. Ozoe, Numerical computation for the melt convection of the model system of continuous steel casting with various magnetic fields, ISIJ Internat., 43, 907914,2003.

5. R. Sekar, G. Vaidyanathan, R. Hemalatha, and S. Sendhilnathan, Effect of sparse distribution pores in a Soret-driven ferro thermohaline convection, J. Magnetism Magnetic Materials,302, 20-28,2006.

6. A. J. Chamkha and H. Al-Naser, Hydromagnetic double-diffusive convection in a rectangular enclosure with uniform side heat and mass fluxes and opposing temperature and concentration gradients, Int. J. Thermal Sciences, 41, 936-948, 2002. 
7. A. Raptis, and J. Vlahos, Unsteady hydromaguetic free convective flow through a porous medium, Lett. Heat Mass Transfer, 9, 56-64,1982.

8. M. Bourich, M. Hasnaoui, A. Amahmid, M. Er-Raki and M. Mamou, Analytical and numerical study of combined effects of a magnetic field and an external shear stress on soret convection in a horizontal porous enclosure, Numerical Heat Transfer, Part A, 54, 1042-1060, 2008.
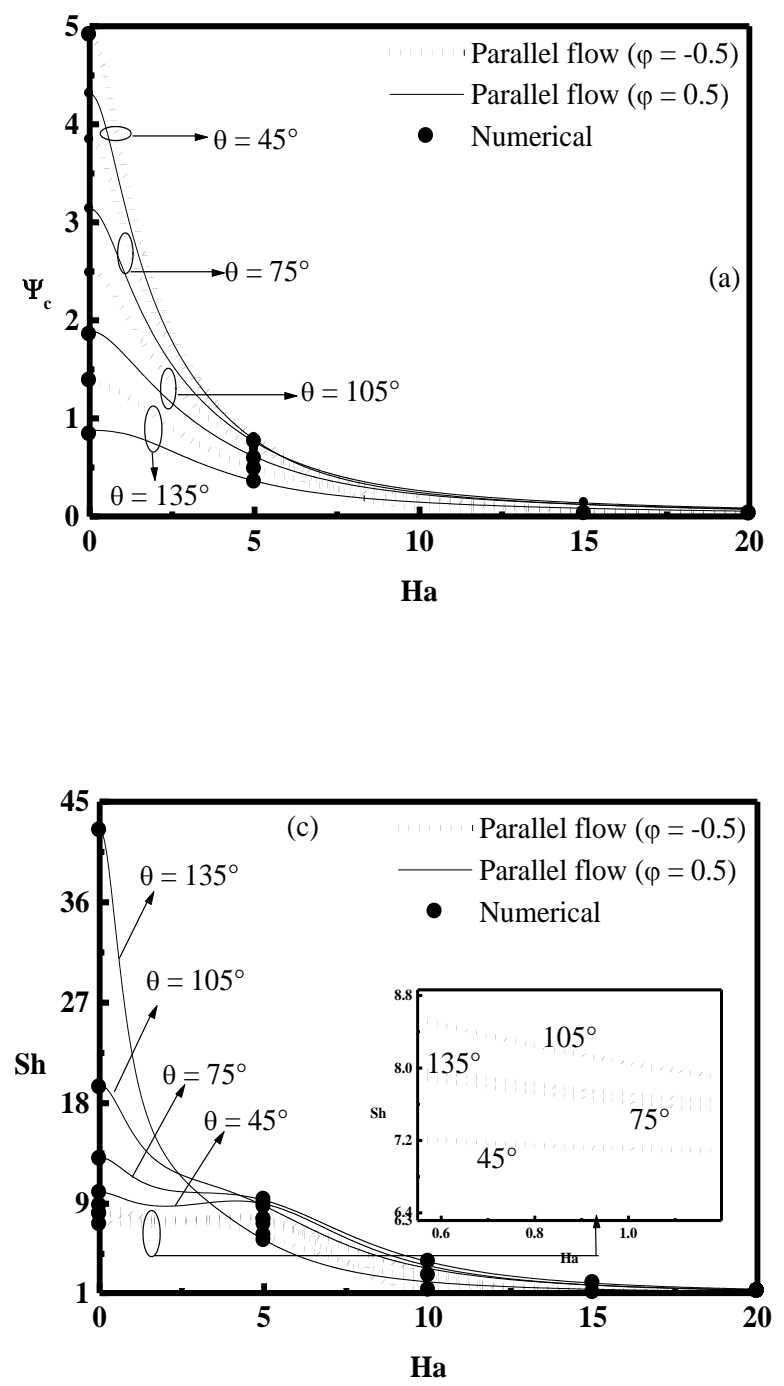
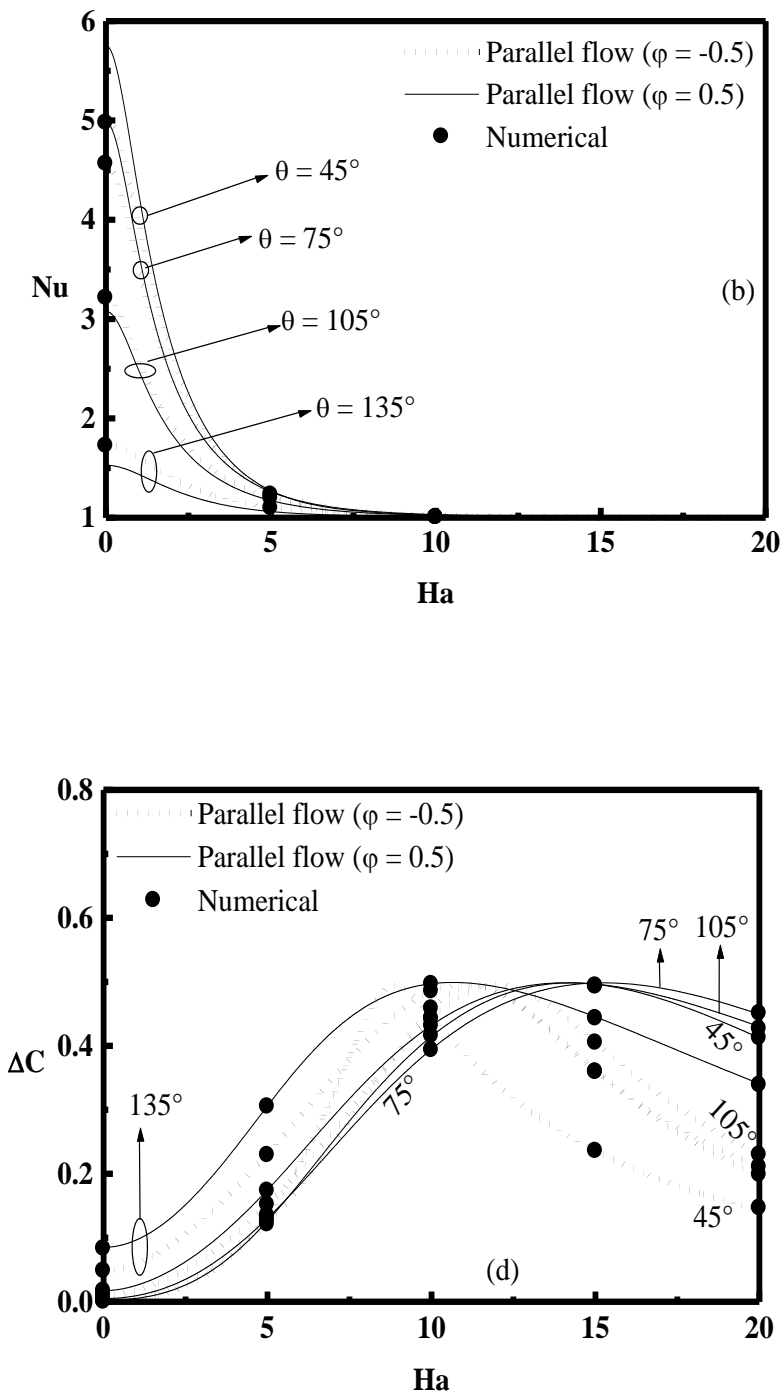

Fig. 3. Effect of Ha on $\Psi_{c}$ (a), $\mathrm{Nu}(\mathrm{b}), \mathrm{Sh}$ (c) and $\Delta \mathrm{C}$ (d) for

$$
\text { Le }=10 \text { and } R_{T}=200 \text {. }
$$

